\title{
Caracterización del ruido de las operaciones de una empresa de fabricación y comercialización de envases de vidrio hidrolítico tipo 1
}

\author{
Noise characterization of the operations of a manufacturing and marketing \\ company of type 1 hydrolytic glass containers
}

\author{
Hermes Jilmer Cano Arratea ${ }^{1}$
}

Recibido: 07/09/2020 - Aprobado: 01/02/2021 - Publicado: 18/06/2021

\begin{abstract}
RESUMEN
La presente investigación realizó un estudio sobre exposición a dosis de ruido a trabajadores en una empresa de fabricación y comercialización de envases de vidrio hidrolítico tipo 1. El objetivo fue caracterizar los perfiles de exposición de los GES por comparación de los resultados con el LMP. La población del estudio está conformada por todos los trabajadores expuestos a ruido, población es 103 y la muestra de 45, la metodología usada es la peor condición (Associates in Acoustics, 2009) y según las directivas de la AlHA. Los resultados se analizaron de acuerdo con dos indicadores, valor máximo para muestras menores a 6 y en caso evaluaciones mayores se analizaron bajo el UCL, obteniendo como resultados 9 Grupos de GES, de las cuales el $89 \%$ presenta un nivel de exposición sin protección auditiva mayor al LMP.
\end{abstract}

Palabras clave: Evaluación de la exposición; audición; conservación de la audición; monitoreo; ruido.

\begin{abstract}
The present investigation carried out a study on exposure to noise doses to workers in a company that manufactures, and markets hydrolytic glass containers type 1. The objective is to characterize the exposure profiles of the GES by comparing the results with the LMP. The study population is made up of all workers exposed to noise and the population of 103 and a sample of 45, the methodology used is that of the worst condition and according to the AlHA guidelines. The results were analyzed according to two indicators, maximum value for samples smaller than 6 and in case of higher evaluations it was analyzed under the UCL, obtaining as results 9 groups of GES, of which $89 \%$ present a level of exposure without hearing protection greater than the LMP.
\end{abstract}

Keywords: Exposure assessment; hearing; hearing conservation; monitoring; noise.

\footnotetext{
1 Ingeniero en Higiene y Seguridad Industrial. Maestría en Gestión Integrada de Seguridad, Salud Ocupacional y Medio Ambiente. Universidad Nacional Mayor de
} San Marcos, Facultad de Ingeniería Geológica, Minera, Metalúrgica y Geográfica. Lima, Perú. E-mail: hs.j..cano@gmail.com 


\section{INTRODUCCIÓN}

En el Perú contamos con una empresa especializada en la fabricación y comercialización de envases de vidrio hidrolítico tipo 1, tales como ampollas, frascos viales entre otros (Vitrum, 2020). Dado que sus operaciones se realizan en su sede en Lima, las principales fuentes de ruido ocupacional son los motores de sistemas de inyección y extracción de aire. Los trabajadores realizan trabajos directamente en las máquinas ampolleteras y con los productos recepcionados del mismo.

\subsection{El perfil de exposición al sonido}

El perfil de exposición se puede definir como la estimación de la intensidad de la exposición y como puede variar en el tiempo para cada Grupo de Exposición Similar GES (AIHA, 2010).

El perfil de exposición al sonido es una aplicación estructurada organizacionalmente del procedimiento basado en tareas y puede ser una metodología confiable y verificable para estimar la exposición al sonido del trabajador que permite el uso óptimo de los datos recopilados en el campo (Hager, 1998).

Los Grupos de Exposición Similares (GES), son grupos de trabajadores que poseen un perfil general similar de exposición al (los) agente(s) bajo estudio, debido a la similitud y frecuencia con que ejecutan la actividad, los materiales y procesos con los cuales trabajan, y la manera similar como llevan a cabo la actividad (AIHA, 2010).

"Muchas veces se usa la palabra ruido para describir el sonido no deseado", asimismo desde un punto de vista mecánico "El sonido generalmente se define como las fluctuaciones en la presión por encima y por debajo de la presión ambiental de un medio que tiene elasticidad y viscosidad. El medio puede ser un sólido, un líquido o un gas (Associates in Acoustics, 2009).

El rango de audición humana aceptado se extiende desde $20 \mathrm{~Hz}$ hasta $20.000 \mathrm{~Hz}$.

El nivel de sonido continuo equivalente, expresado como Leq, T, se usa para cuantificar el nivel de presión sonora promedio para un cierto período de medición. En la ecuación $\mathrm{N}^{\circ} 1$ se muestra de manera matemática (Associates in Acoustics, 2009).

$$
\begin{aligned}
& \text { LAeqT }=10 \log \left(\frac{1^{t 1}}{T t 1} \int P\left[\frac{A(t)}{P o}\right]^{2}\right) d t \ldots \ldots . \text { Ec. } 1 \\
& \mathrm{P}_{\mathrm{A}}=\text { Presión Acústica } \\
& \mathrm{P}_{0}=\text { Presión de Referencia }
\end{aligned}
$$

El Resolución Ministerial N³75-2008-TR establece los estándares nacionales para ruido ocupacional en relación con el valor del nivel diario de exposición al ruido ponderado A, LEX,8h, el cual se calcula a partir de la ecuación $\mathrm{N}^{\circ} 2$.

$$
\text { LEx, } 8 \mathrm{~h}=\mathrm{LP}, \mathrm{A} \text {, eqte }+10 \log \int\left(\frac{T e}{T_{0}}\right) d t \ldots \text { Ec. } 2
$$

$\mathrm{L}_{\mathrm{p}, \mathrm{AeqTe}}$ es el nivel de presión sonora equivalente ponderado A para la duración efectiva de la jornada laboral Tabla 1).

$\mathrm{T}_{\mathrm{e}}$ es la duración efectiva de la jornada laboral (ver

$\mathrm{T}_{\mathrm{o}}$ es la duración de referencia $\mathrm{T}_{0}=8 \mathrm{~h}$

Tabla 1. Límites máximos permisibles de exposición a ruido

\begin{tabular}{cc}
\hline Nivel de ruido en escala "A", dB(A) & Tiempo de exposición \\
\hline 100 & $1 / 4$ hora/ día \\
97 & $1 / 2$ hora/ día \\
94 & 1 hora/ día \\
91 & $1 \frac{1}{2}$ horas/ día \\
88 & 4 horas/ día \\
85 & 8 horas/ día \\
84.5 & 9 horas/ día \\
83 & 12 horas/ día \\
82 & 16 horas/ día \\
\hline
\end{tabular}

Fuente: RM-375-2008-TR (Ministerio de Trabajo y Promoción del Empleo (MTPE), 2008)

Para fines del estudio se ha elaborado una escala en función del Límites máximos permisibles (LMP), tal como se muestra en la Figura 1.

\begin{tabular}{|c|l|}
\hline Nivel de riesgo & \multicolumn{1}{|c|}{ Nivel de exposición } \\
\hline Bajo & Exposición a ruido < Nivel de Acción* \\
\hline Medio & Nivel de Acción $\leq$ Exposición a ruido < LMP \\
\hline Alto & LMP $\leq$ Exposición a ruido \\
\hline
\end{tabular}

Figura 1. Semaforización de niveles de exposición. Fuente: Elaboración propia

Nota: Nivel de Acción es el 50\% del LMP considerando una tasa de cambio de $3 \mathrm{~dB}$. Para un LMP de $85 \mathrm{~dB}$, el Nivel de Acción será $82 \mathrm{~dB}$.

\section{MÉTODOS}

\subsection{Población de estudio}

La evaluación se realizó con dosímetros de ruido, los cuales fueron aportados por cada trabajador durante el $70 \%$ de su jornada laboral, que es igual a 8 horas diarias.

La muestra del estudio objetivo estuvo constituida por 45 trabajadores que fueron agrupados en nueve (09) GES, en la tabla 2, se muestra los puestos que conformaron el estudio.

El Programa de Monitoreo Ocupacional del año 2019, contempló la evaluación de nueve (09) GES, los cuales se muestra sus respectivos códigos y cantidad de muestras evaluadas. 
Para elección de muestra, el estudio consideró dos criterios 1) método de la peor condición, el cual consiste en realizar mediciones menores a seis (06) y escoger el valor más alto obtenido como representativo, considerando el criterio de mayor protección para el trabajador, 2) Recomendación de la Asociación Americana de Higiene Industrial (AIHA, 2010), que considera que "La línea base mínima debe constar de un mínimo de seis muestras. En la Tabla 3, se puede observar la muestra (n) para cada población (N) de los GES (Ver Figura 2).

\section{RESULTADOS}

Ver Tabla 4 y Figura 3.

Tabla 2. Puestos que conforman los GES con sus respectivas poblaciones

\begin{tabular}{lllc}
\hline \multicolumn{1}{c}{ Código GES } & \multicolumn{1}{c}{ Nombre del GES } & \multicolumn{1}{c}{ Puestos que conforman el GES } & $\mathbf{N}^{\circ}$ de personas (N) \\
\hline CGES-001 & Almaceneros & Almacenero & 6 \\
CGES-002 & Analistas de control de calidad & Analista & 11 \\
CGES-003 & Artes gráficas & Artes gráficas & 2 \\
CGES-004 & Operador de servicios generales & Carpintero & 2 \\
CGES-006 & Mecánicos-Eléctricos & Mecánicos-Eléctricos & 7 \\
CGES-011 & Operadores de Producción & Operador & 20 \\
CGES-012 & Reguladores de Producción & Regulador & 9 \\
CGES-014 & Verificadores & Revisora & 38 \\
CGES-015 & Jefes de planta & Supervisor & 8 \\
\hline
\end{tabular}

Fuente: Elaboración propia

Tabla 3. La cantidad de muestras elegida

\begin{tabular}{llll}
\hline \multicolumn{1}{c}{ Descripción del GES } & \multicolumn{1}{c}{ Código del GES } & N & $\mathrm{n}$ \\
\hline Almaceneros & CGES-001 & 6 & 2 \\
Analistas de control de calidad & CGES-002 & 11 & 2 \\
Artes gráficas & CGES-003 & 2 & 2 \\
Operador de servicios generales & CGES-004 & 2 & 1 \\
Mecánicos-Eléctricos & CGES-006 & 7 & 5 \\
Operadores de Producción & CGES-011 & 20 & 10 \\
Reguladores de Producción & CGES-012 & 9 & 10 \\
Verificadores & CGES-014 & 38 & 10 \\
Jefes de planta & CGES-015 & 8 & 3 \\
\hline
\end{tabular}

Fuente: Elaboración propia

Tabla 4. Nivel diario de exposición al ruido ponderado A, LEX,8h, para cada GES y con indicador correspondiente según el criterio de elección de muestra

\begin{tabular}{|c|c|c|c|c|c|}
\hline \multirow{2}{*}{ Descripción del GES } & \multirow{2}{*}{ Código del GES } & \multirow{2}{*}{$\mathrm{n}$} & \multicolumn{2}{|c|}{ Nivel de exposición por GES, sin protección auditiva } & \multirow{2}{*}{ Nivel de Riesgo } \\
\hline & & & Max & UCL & \\
\hline Almaceneros & CGES-001 & 2 & 81 & N.A & Bajo \\
\hline Analistas de control de calidad & CGES-002 & 2 & 85.5 & N.A & Alto \\
\hline Artes gráficas & CGES-003 & 2 & 88.5 & N.A & Alto \\
\hline Operador de servicios generales & CGES-004 & 1 & 100.8 & N.A & Alto \\
\hline Mecánicos-Eléctricos & CGES-006 & 5 & 88 & N.A & Alto \\
\hline Operadores de Producción & CGES-011 & 10 & N.A & 85.3 & Alto \\
\hline Reguladores de Producción & CGES-012 & 10 & N.A & 92.8 & Alto \\
\hline Verificadores & CGES-014 & 10 & N.A & 85.3 & Alto \\
\hline Jefes de planta & CGES-015 & 3 & 89.2 & N.A & Alto \\
\hline \multicolumn{3}{|c|}{ Límite Máximo Permisible (LMP) para $8 \mathrm{Hrs}$} & \multicolumn{2}{|c|}{85.0} & \\
\hline \multicolumn{3}{|l|}{ Nivel de Acción (NA) } & \multicolumn{2}{|c|}{82.0} & \\
\hline
\end{tabular}

Fuente: Elaboración propia 


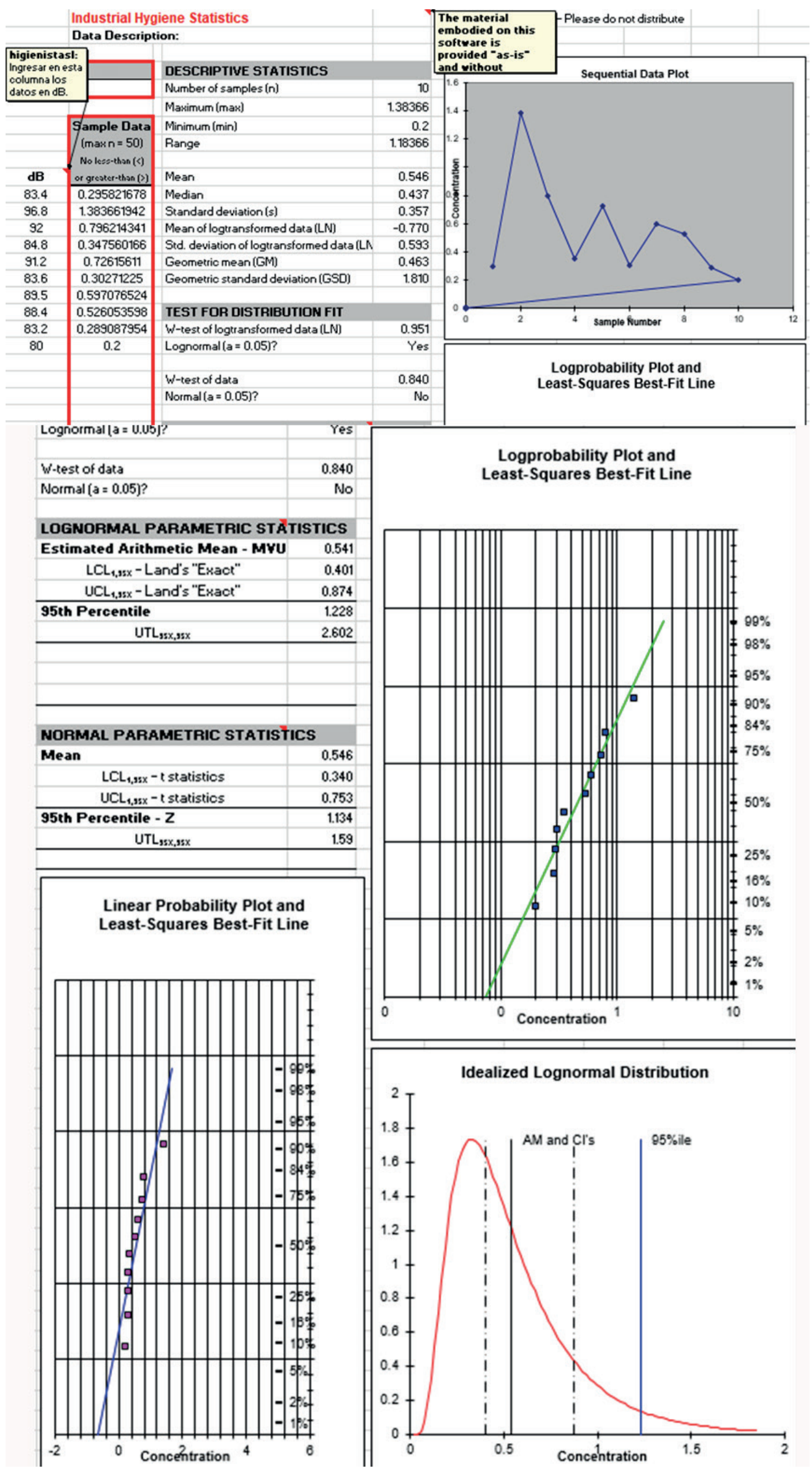

Figura 2. Herramienta usada para cálculo de. Límite de confianza superior (UCL). 


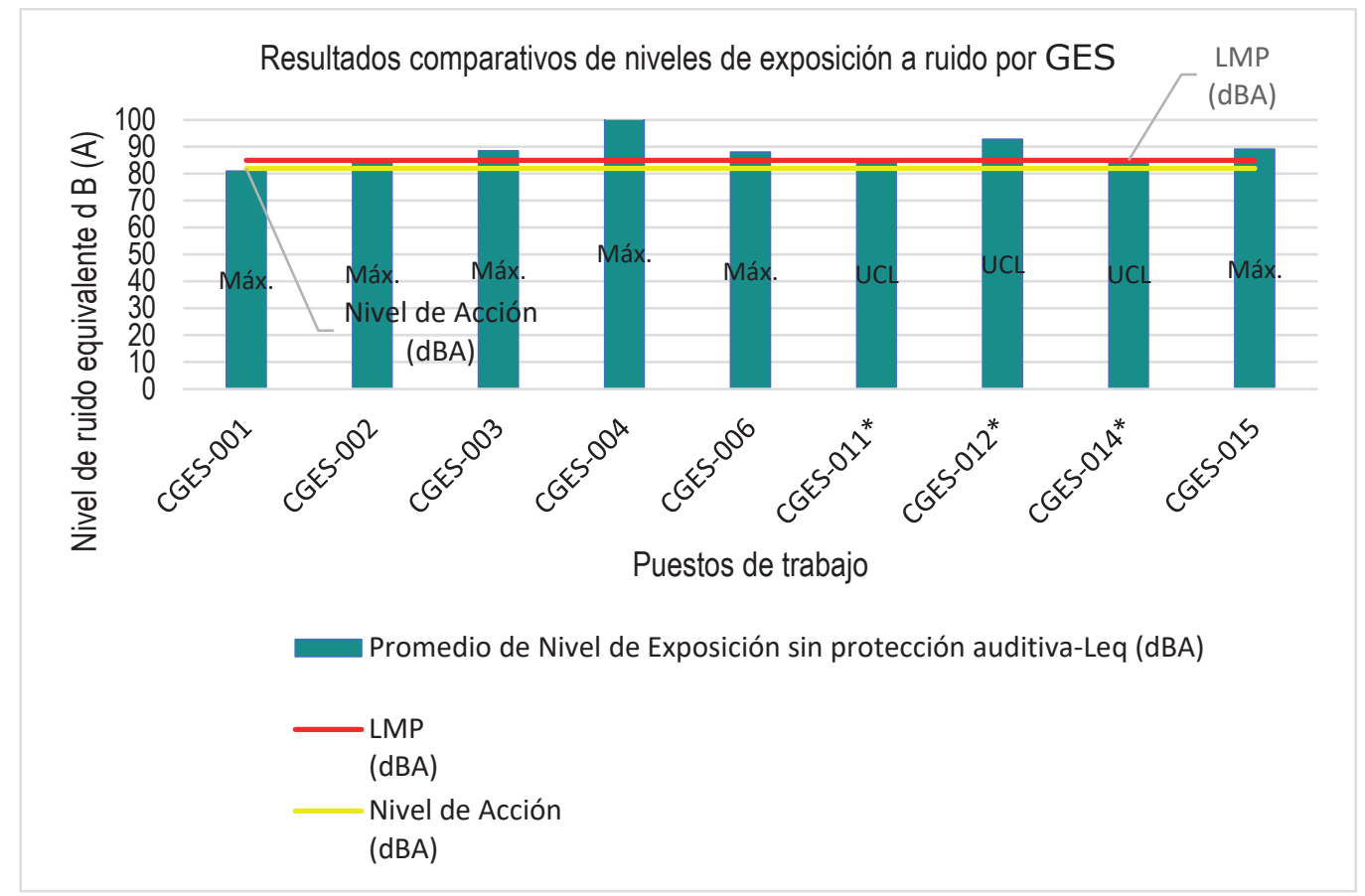

Figura 3. Resultados comparativos de niveles de exposición a ruido por GES. Fuente: Elaboración propia

\section{DISCUSIÓN}

\subsection{Grupos de exposición similar (GES)}

Como se muestra en la Tabla 2, se agrupó a los puestos en nueve (09) GES, estos conformados por Almaceneros (CGES-001), Analistas de control de calidad (CGES002), Artes gráficas (CGES-003), Operador de servicios generales (CGES-004), Mecánicos-Eléctricos (CGES006), Operadores de Producción (CGES-011), Reguladores de Producción (CGES-012), Verificadores (CGES-014) y Jefes de planta (CGES-015).

\subsection{Resultado de las evaluaciones de dosimetrías}

Los resultados se muestran en la Tabla 4, asimismo se muestra la semaforización de niveles de exposición conforme la Figura 1.

En la Figura 3, se realizó una comparación entre el nivel de exposición ponderado sin protección auditivaLEX, 8h (dBA) con el Límite Máximo permisible (LMP), asimismo cada barra se indica el resultado reportado, es decir el máximo (Max) o el límite de confianza superior (UCL). Para los GES CGES-011, CGES-012 y CGES014 se comparó el valor UCL y en los 3 casos supera el LMP, en los demás GES se concluyó con el valor máximo registrándose valores superiores al LMP.

\subsection{Análisis de resultados}

El análisis de resultado se realizó conforme al criterio de elección de la muestra, para aquellos GES que presentan una cantidad de muestras menores a seis se concluye bajo la metodología de la peor condición, es decir con valor máximo y para los grupos de exposición similar que superen la cantidad de muestras igual a seis, se analizó los resultados bajo el indicador de límite de confianza superior al 95\% (UCL, 95\%).

El procedimiento “exacto" de Land se usó para calcular el límite de confianza superior (UCL1, 95\%), esto consiste en introducir nivel de exposición ponderado, el software convierte en forma automática a Pascales cuadrado $(\mathrm{Pa})^{2}$ y analiza los resultados bajo una Estadísticas Paramétricas Normal o Log-Normales, asimismo sugiere que parámetro estadístico se debería usar

\section{CONCLUSIONES}

- Las fuentes de ruido para los GES evaluados están conformados por máquina de moldeo viales 3BS30-10/2 y 9 motores de extracción de potencia 2HP, Marca Weg., 2 motores de extracción de potencia $2 \mathrm{HP}$, Marca Weg, asimismo tiene una influencia importante por las fuentes de la zona de aire de inyección ubicados en la azotea conformados por 2 motores de $10 \mathrm{Kw}$.

- $\quad$ El 89\% de los Grupos de Exposición Similar (GES) evaluados, presenta un nivel de exposición sin protección auditiva mayor al Límite Máximo Permisible (LMP). Este panorama resulta bastante desfavorable y evidencia que 08 de los GES tienen categoría de alto riesgo, lo que supone un trabajo de riesgo y probablemente, a futuro cercano, causará daño en la salud de los trabajadores.

- Los grupos de exposición similar, evaluados, permiten una oportunidad de mejora, en caso 
de los GES reportados con valores máximos tener en cuenta las evaluaciones anuales año tras año para generar los perfiles de exposición y en caso de los GES reportados bajo el indicador UCL, entendiéndose que el nivel ponderado sin protección auditiva-LEX, 8h (dBA) con mayor cercanía al LMP son los de mayor interés se está considerando la aplicación del método NIOSH en la próximas elección de muestras para aquellos valores de UCL - RUIDO que estuvieron en el rango siguiente: $0.5 \mathrm{LMP} \leq \mathrm{UCL} \leq 2 \mathrm{LMP}$. Así como la elaboración de estándar de Equipos de protección Auditiva homogéneo para cada GES, a fin de considerarse en los cálculos de riesgos residuales de ruido en los próximos estudios.

- En el cálculo de UCL, para el GES Operadores de Producción (CGES-011), Reguladores de Producción (CGES-012) y verificadores (CGES014), se obtuvo una desviación geométrica estándar la variabilidad de baja el cual indica que existe poca variabilidad entre los resultados, asimismo el UCL quiere decir que se tiene el 95\% de confianza que la proporción de individuos con promedio de exposición por debajo del LMP es menos del $5 \%$.

- En los próximos estudios se debe considerar factores sociales, laborales y biológicos, diferenciados en función del sexo, incorporando la dimensión de género en la evaluación y prevención de los riesgos en la salud laboral, conforme al principio de prevención de la Ley $\mathrm{N}^{\circ} 29783$, Ley de Seguridad y Salud en el Trabajo.

- De acuerdo con la Norma Básica de Ergonomía y de Procedimiento de Evaluación de Riesgo R.M. N N $^{\text {375-2008-TR (Ministerio de Trabajo }}$ y Promocion del Empleo, 2008) los Grupos de Exposición Similar (GES) estén expuestos a niveles por encima de $85 \mathrm{~dB}(\mathrm{~A})$, no podrán estar expuestos un tiempo mayor a 8 horas.

\section{AGRADECIMIENTOS}

Para mi familia, compañeros y a nuestro querido pueblo, que me ayudaron a iniciar, persistir y culminar el presente trabajo.

A mi asesora la Mg. Nora Rosa Concepción Malca Casavilca, mi aprecio y un gran reconocimiento por su capacidad de colaboración y contribución en el asesoramiento.

\section{REFERENCIAS}

AIHA. (2010). La estrategia para la evaluación de la exposición ocupacional. In American Industrial Hygiene Association. American Industrial Hygiene Association. https://es.scribd. com/document/421892766/Evaluacion-OcupacionalAIHA-pdf

Associates in Acoustics, I. B. (2009). Manual del Estudiante: ruido, medición y sus efectos. Associates in Acoustics, Inc, BP International Limited and the University of Wollongong, 330. https://www.ohlearning.com/Files/Student/JC22 v1-0 09Apr10 W503 Manual del estudiante 1.pdf

Hager, L. D. (1998). Sound Exposure Profiling: A Noise Monitoring Alternative. AIHAJ, 59(6), 414-418. https://doi. org/10.1202/0002-8894(1998)059<0414:sepanm>2.0.co;2

Ministerio de Trabajo y Promocion del Empleo. (2008). Resolución Ministerial 375-2008 TR Norma Basica de Ergonomia y de Procedimiento de Evaluación de Riesgo Disergonómico. El Peruano, 1-17. www.mintra.gob.pe,

Ministerio de Trabajo y Promoción del Empleo (MTPE). (2008). Resolución Ministerial $N^{\circ}$ 375-2008-TR. Normas Legales Diario El Peruano. https://www.gob.pe/institucion/mtpe/ normas-legales/394457-375-2008-tr

Vitrum, A. (2020). Vidrio Hidrolitico tipo I. Amfa Vitrum S.A. https://www.amfa.com.pe/hidroliticol.php 